\title{
Induction of the LRP16 gene by estrogen promotes the invasive growth of Ishikawa human endometrial cancer cells through the downregulation of E-cadherin
}

\author{
Yuan Guang Meng ${ }^{1}$, Wei Dong $\mathrm{Han}^{2}$, Ya Li Zhao², Ke Huang ${ }^{1}$, Yi Ling $\mathrm{Si}^{2}$, Zhi Qiang $\mathrm{Wu}^{2}$, Yi Ming $\mathrm{Mu}^{3}$ \\ ${ }^{1}$ Department of Obstetrics and Gynecology, Chinese PLA General Hospital, 28 Fuxing Road, Beijing 100853, China; ${ }^{2}$ Department \\ of Molecular Biology, The Institute of Basic Medicine, Chinese PLA General Hospital, 28 Fuxing Road, Beijing 100853, China; \\ ${ }^{3}$ Department of Endocrinology, Chinese PLA General Hospital, 28 Fuxing Road, Beijing 100853, China
}

LRP16 was previously identified as an estrogen-induced gene in breast cancer cells. The responsiveness of LRP16 to estrogen and its functional effects in endometrial cancer (EC) cells are still unclear. Here, we show that the mRNA level and promoter activity of the LRP16 gene were significantly increased by $17 \beta$-estradiol (E2) in estrogen receptor $\alpha(\mathrm{ER} \alpha)$-positive Ishikawa human EC cells. Although the growth rate of Ishikawa cells was not obviously affected by ectopic expression of LRP16, the results of a Transwell assay showed an approximate one-third increase of the invasive capacity of LRP16-overexpressing cells. As a result of molecular screening, we observed that the expression of E-cadherin, an essential adhesion molecule associated with tumor metastasis, was repressed by LRP16. Further promoter analyses demonstrated that LRP16 inhibited E-cadherin transactivation in a dose-dependent manner. However, the inhibition was abolished by estrogen deprivation, indicating that the downregulation of E-cadherin transcription by LRP16 requires ER $\alpha$ mediation. Chromatin immunoprecipitation analyses revealed that the binding of ER $\alpha$ to the E-cadherin promoter was antagonized by LRP16, suggesting that LRP16 could interfere with ER $\alpha$-mediated transcription. These results suggest that the upregulation of LRP16 by estrogen could be involved in invasive growth by downregulating E-cadherin in human ECs.

Keywords: LRP16, ER $\alpha$, E-cadherin, Ishikawa, invasiveness

Cell Research (2007) 17:869-880. doi: 10.1038/cr.2007.79; published online 25 September 2007

\section{Introduction}

Estrogens play crucial roles in the normal physiology of a variety of tissues, including those of mammary glands, reproductive tract, central nervous system and skeleton [1]. Estrogen mediates its biological effects through two receptors $\alpha$ and $\beta$ (ER $\alpha E R \beta)$, which function as liganddependent transcriptional activators [2]. The subsequent transactivation of ER target genes occurs through the direct

\footnotetext{
Correspondence: Wei Dong Han

Tel/Fax: +86-10-6693-7516

E-mail: hanwdrsw69@yahoo.com

Received 22 January 2007; revised 1 April 2007; accepted 9 May 2007; published online 25 September 2007
}

binding of liganded ER to classical estrogen response elements (ERE) in gene promoters or indirect binding through interactions with other transcriptional factors such as Ap1, $\mathrm{Sp} 1$ or nuclear factor $\kappa \mathrm{B}[3-5]$. The biological functions downstream of ER result from the altered expression of direct transcriptional targets as well as secondary effects that are mediated by the biological activities of direct targets.

The endometrium is a classical hormone-dependent tissue and most of the endometrial adenocarcinomas, which represent over $80 \%$ of endometrial cancers (ECs), are hormone-dependent tumors. Elevated estrogen levels or unopposed estrogen exposure has been linked to the development and progression of ECs [6,7], hence it is important to delineate the molecular mechanisms that underlie the roles of estrogen in endometrial carcinogenesis. 
EC cell lines have been used as models for understanding the mechanisms associated with 17 $\beta$-estradiol (E2)-induced gene expression and for the development of antiestrogenic and anticarcinogenic agents for treating this disease [8]. Among these ER $\alpha$-positive EC cell lines, Ishikawa cells, which are derived from the well-differentiated human endometrial adenocarcinoma, are an extensively used model [9-11].

E-cadherin is a major component of adherent junctions in vertebrates and is essential for establishing tight physical cell-cell adhesion $[12,13]$. Transcriptional inactivation of the E-cadherin gene has been widely linked to the acquisition of the invasive phenotype for many epithelial tumors including ECs $[14,15]$. In addition to the promoter hypermethylation of the E-cadherin gene, overexpression of transcriptional repressors such as Snail and Twist is an alternative mechanism for the decrease of E-cadherin gene expression in breast tumors $[16,17]$. Indeed, there is evidence to show that the expression level of the E-cadherin gene is directly linked to ER $\alpha$ status in human breast cancer cells $[18,19]$. But for ECs, the inactivation mechanism of E-cadherin is poorly understood.

The human LRP16 gene, which was originally isolated from peripheral lymphocyte cells by our group [20], contains an open reading frame for a protein of 325 amino acids. LRP16 protein mainly localizes in the nuclei of several cell lines [21] and contains a Macro_Appr_pase-

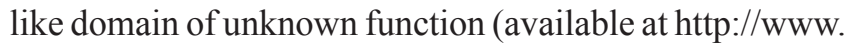
ncbi.nlm.nih.gov/Structure/cdd/cdd.shtml). Previous studies have demonstrated that E2 upregulates the promoter activity of LRP16 through Sp1-mediated pathways in MCF-7 breast cancer cells [22, 23]. LRP16 overexpression in MCF-7 significantly stimulates cell proliferation [22]. The expression level of LRP16 mRNA was detected to be higher in $40 \%$ of primary breast cancers, and the clinicopathological characteristics, such as ER/PR-status, tumor diameter and the involvement of axillary lymphoid nodes, were tightly linked with LRP16 mRNA expression level [24]. These findings imply that LRP16 might have an important role in carcinogenesis and/or in the progression of hormone-dependent cancers, and led us to explore the regulatory mechanisms of LRP16 expression and its possible function in EC.

In this study, we focus on the regulation of LRP16 expression by E2 and its functional significance in Ishikawa human EC cells. Our results document a novel molecular pathway of ER $\alpha /$ LRP16/E-cadherin and reveal a role for LRP16 in promoting cancer cell invasion in vitro.

\section{Materials and Methods}

\section{Cell culture}

All cell lines were purchased from the American Type Culture
Collection (ATCC) (Rockville, MD, USA) and were cultured according to the manufacturer's instructions. pL374 and pL668 are MCF-7 cell lines that stably express LRP16-SiRNA and pLGFPi is an MCF-7 cell line that stably expresses control siRNA. These were previously established in our laboratory using a retrovirus-mediated hairpin-siRNA expressing system. The endogenous LRP16 inhibition rate in the above cell lines was $90 \%, 60 \%$ and $0 \%$, respectively [25]. These cells were cultured in DMEM that was supplemented with puromycin $(1 \mu \mathrm{g} / \mathrm{ml})$. Ishikawa and T47D cells were cultured in DMEM supplemented with $10 \%$ fetal calf serum (FCS) and MDAMB-231 cells were maintained in L15 medium supplemented with $10 \%$ FCS. 17 $\beta$-Estradiol (E2) was purchased from Sigma (St Louis, MO, USA). The pure estrogen antagonist ICI 182780 was provided by Dr Qinong Ye at the College of Military Medicine Science of China. Phenol-free medium was purchased from the Institute of Basic Medicine, Xiehe Hospital (Beijing, China). Steroid-deprived FCS was prepared as described previously [23].

\section{Plasmids}

The human LRP16 full-length cDNA was cloned into the pcDNA3.1 expression vector as described previously [22]. Mammalian expression plasmids for estrogen receptor $\alpha(\mathrm{pS} 5 \mathrm{G}-\mathrm{hER} \alpha)$ were provided by Professor Hajime Nawata (Kyushu University, Fokuoka, Japan). The E-cadherin expression vector pcDNA3.1-Ecad was provided by $\mathrm{Dr} \mathrm{Xu} \mathrm{Li}$ at the University of Tennessee, USA. The luciferase reporter gene construct (E-cadK1-Luc) containing the E-cadherin sequence was provided by Dr Eric R Fearon at the University of Michigan, USA [26]. The luciferase reporter pGL-S included the fragment of -676 to $-24 \mathrm{bp}$ from the LRP16 upstream regulatory region, which contains an ERE half site (ERE1/2)/GC-rich Sp1 binding site as described previously [24].

\section{Transfection}

Expression vector pcDNA3.1-LRP16 or the blank pcDNA3.1 was transfected into Ishikawa cells using the Superfect transfection reagent (Qiagen, Hilden, Germany) according to the manufacturer's instructions. Two days after transfection, the cells were treated with G418 (Gibco BRL, Grand Island, NY, USA) at $1 \mathrm{mg} / \mathrm{ml}$ for 2 weeks and were then continuously cultured with $0.5 \mathrm{mg} / \mathrm{ml} \mathrm{G} 418$.

Ishikawa cells were seeded in 10-cm culture dishes and grown to $90 \%$ confluence until transfection. siRNA duplexes were transfected using Lipofectamine 2000 according to the manufacturer's recommendations (Invitrogen). siRNA oligonucleotides were chemically synthesized by Shanghai GeneChem Co., Ltd (Shanghai, China). The sequence of LRP16-siRNA 374 (sense strand, 5'- GCAGCGGGAGGAACAUUCATT-3') was reported previously [25]. The unrelated siRNA sequence (sense strand, 5'-UUCUCCGAACGUGUCACGUTT-3') was used as control. The siRNA duplex was transfected in each dish to give a final concentration of $50 \mathrm{nM}$. Cells were harvested $48 \mathrm{~h}$ after transfection for immunoblotting analyses and Transwell assays.

\section{RNA isolation and northern analysis}

Total RNA was isolated by the acid guanidium thiocyanate-phenol chloroform method using TriBlue reagent (Biotec Co., Beijing, China). Twenty micrograms of total RNA was electrophoresed through a $1 \%$ agrose gel containing formaldehyde and was transferred to a Hybond $\mathrm{N}^{+}$membrane (Amersham, Buckinghamshire, UK). The membranes were hybridized using the following probes labeled 
with $\left[\alpha{ }^{32} \mathrm{P}\right] \mathrm{dCTP}$ by random priming: 550-bp fragment of LRP16 (432 to 981 bp, NM_014067), $1.9 \mathrm{~kb}$ of E-cadherin (339 to 2240 bp, NM 004360), 573 bp of Snail (125 to 697 bp, NM 005985), 722 bp of Slug (249 to 970 bp, NM_003068), 465 bp of SIP1 (498 to $962 \mathrm{bp}, \mathrm{NM} \_04785$ ) and $515 \mathrm{bp}$ of $\beta$-actin (457 to $971 \mathrm{bp}$, NM_001101).

\section{Antibodies, immunoblotting and immunohistochemistry}

Antiserum against human LRP16 protein was generated in rabbits using pRSET-C-expressed LRP16 antigen (amino acids 83-324). The other antibodies utilized were E-cadherin (BD Transduction, USA), MMP2, MMP9, CD44 and $\beta$-actin (Santa Cruz Biotechnology, CA, USA). For immunoblotting, cell lysates were electrophoresed by SDS-PAGE using 10\% acrylamide gels and blotted onto PVDF membranes (Amersham). Blots were probed with the primary antibodies, washed and then incubated with horseradish peroxidase-labeled secondary antibodies (Santa Cruz), and binding was detected using enhanced chemiluminesence.

For immunohistochemistry, cells cultured on cover slips were rinsed twice with PBS and fixed with ice-cold methanol for $10 \mathrm{~min}$. The antibody dilution of E-cadherin was 1:200. Detection with the PV-9000 system (Santa Cruz) for immunoperoxidase staining followed the manufacturer's recommendations. Cells were counterstained with hematoxylin before mounting for microscopy.

\section{Cell proliferation assay}

To measure cell growth rates, cells were seeded at $1 \times 10^{4}$ per well in 24-well plates. After cells were attached to the wells, the medium was replaced with $1 \mathrm{ml}$ of fresh DMEM with $1 \%$ FBS, and was changed every 2 days. Cells from three dishes were trypsinized and counted each day. Each experiment was repeated three times. Based on the counted cell numbers at different time points, a cell proliferation curve was generated.

\section{Luciferase reporter assays}

Cells of $50 \%$ confluence in $35-\mathrm{mm}$ dishes were transfected using the Superfect reagent. $0.25 \mu \mathrm{g}$ of the E-cadherin promoter gene construct, or the LRP16 promoter gene construct, was co-transfected with 1 ng of pRL-SV40 (Promega, WI, USA) per well. Cell extracts were prepared $42 \mathrm{~h}$ after transfection, and the luciferase activity was measured using the Dual-Luciferase Reporter Assay System (Promega). All experiments were performed in triplicate.

\section{Boyden chamber invasion assays (Transwell assays)}

Invasion assays were carried out in modified Boyden chambers with 8 - $\mu \mathrm{m}$ pore filter inserts for 24 -well plates (BD Transduction, USA). The surfaces of the filters were coated with $15 \mu$ l of ice-cold Matrigel (15 mg/ml protein; BD Tranduction) for $60 \mathrm{~min}$ at room temperature. Uniformity of the coating was checked by Coomassie blue staining and low-power microscopic observation. The lower chamber was filled with medium containing $10 \%$ serum. Fibronectin $(16 \mu \mathrm{g} /$ chamber $)$ was added as the chemoattractant to the lower chamber. Ishikawa cells $\left(1 \times 10^{5}\right.$ cells/well $)$ were washed with $1 \times$ PBS twice, re-suspended in $200 \mu 1$ of serum-free medium with or without $1 \mu \mathrm{M}$ E2 and then were transferred into the upper chamber. After $24 \mathrm{~h}$ of incubation the filter was gently removed from the chamber, the cells on the upper surface were removed by wiping with a cotton swab, and the cells that invaded the Matrigel and attached to the lower surface were fixed, stained with hematoxylin and eosin (H\&E) and counted in 15 randomly selected microscopic fields $(\times 100)$. Experiments were performed independently at least three times.

\section{Chromatin immunoprecipitation assays}

Ishikawa cells $\left(1 \times 10^{6}\right)$ were grown in 10 -cm tissue culture plates in phenol-red-free DMEM supplemented with $10 \%$ steroid-depleted FCS. After $24 \mathrm{~h}$, the cells were transfected with $10 \mu \mathrm{g}$ of plasmid DNA mixture (1:1:1 for E-cadK1-Luc, pcDNA3.1-LRP16 and pS5G-hER $\alpha$ or E-cadK1-Luc, pcDNA3.1 and pS5G-hER $\alpha$ ) using the Superfect reagent. Forty hours later, the cells were treated with $10 \mathrm{nM}$ E2 for 3 h. Chromatin immunoprecipitation (ChIP) assays were performed according to the protocol for the ChIP assay kit (Upstate Biology, NY, USA). Immunoprecipitation was carried out overnight at $4{ }^{\circ} \mathrm{C}$ with ER $\alpha$ (Santa Cruz) or LRP16 antibody. DNA fragments were purified with a QIAquick Spin Kit (Qiagen). The presence of the target gene promoter sequences in both the input and the recovered DNA immunocomplexes was detected by PCR. The proximal promoter $(-103$ to $+117 \mathrm{bp})$ of E-cadherin was amplified using the following primer set: forward primer, 5'-CGCTGCTGATTGGCTGTGGC-3' and reverse primer, 5'-CGGGGACGCCGAGCGAGGGC-3'.

\section{Statistical analysis}

Experiments were repeated three or more times, and data are expressed as the mean \pm SEM. Statistical differences were determined using the Statview software and Student's $t$-test. $P<0.05$ was considered to be significantly different.

\section{Results}

Hormonal activation of LRP16 gene/gene promoter constructs in Ishikawa cells

Previously published work from our laboratory demonstrated that E2 upregulates the mRNA expression level of the human LRP16 gene in MCF-7 human breast cancer cells [22]. Here, to address whether LRP16 can be induced by E2 in ER $\alpha$-positive human EC Ishikawa cells, cells were cultured with phenol-red-free DMEM supplemented with steroid-stripped FBS for at least 3 days and then treated with $10 \mathrm{nM}$ E2 over a 72-h time period. The total RNA was then extracted and the expression level of LRP16 mRNA was determined by northern blot analysis. As seen in Figure 1A, an approximate three-fold increase of LRP16 mRNA level was observed as early as $3 \mathrm{~h}$ after the addition of E2 and it was retained at the 24-h time point. The elevated expression subsequently decreased with time, but two- and 1.5-fold expression levels were still observed at 48- and 72-h time points. These results are comparable with those reported previously in ER $\alpha$-positive human breast cancer MCF-7 cells [22]. However, although E2 induces LRP16 expression in both MCF-7 and Ishikawa cells, a continued increase can be observed in MCF-7 cells within the time course. To assess the effect of anti-estrogen on the expression level of LRP16 in Ishikawa cells, the selective estrogen antagonist, ICI 182780 , was used at a concentration of 100 $\mathrm{nM}$ to treat proliferating cells. Total RNA over a 72-h time 
course was extracted and northern blot analysis was used to determine the mRNA level. As shown in Figure 1A, treatment with ICI 182780 resulted in a time-dependent decrease of LRP16. These results indicate that the growth media in these experiments (DMEM with 10\% FCS) contained sufficient steroids to provide normal ERa function and the treatment with ICI 182780 blocked the E2 induction of LRP16. These data confirm the estrogen regulation of LRP16 at the mRNA level in Ishikawa cells.

To address the regulatory mechanism of LRP16 expression by E2 in Ishikawa cells, we co-transfected different amounts of the ER $\alpha$ expression vector and the luciferase reporter $\mathrm{pGLS}_{5}$, which includes the LRP16 promoter fragment that was identified to confer the estrogen responsiveness in MCF-7 and HeLa cells as reported previously [23]. The results summarized in Figure 1C show that E2 induces luciferase activity in EC Ishikawa cells, human breast cancer T47D cells and MDA-MB-231 cells. Meanwhile, an increase in the exogenous amount of ER $\alpha$ markedly increased luciferase activity in these three cell lines. These results confirm that E2 upregulates LRP16 expression via $\mathrm{ER} \alpha$ transactivation and that the level of LRP16 expression is dependent on the estrogen activity in several epithelial tumor cells, including Ishikawa cells.
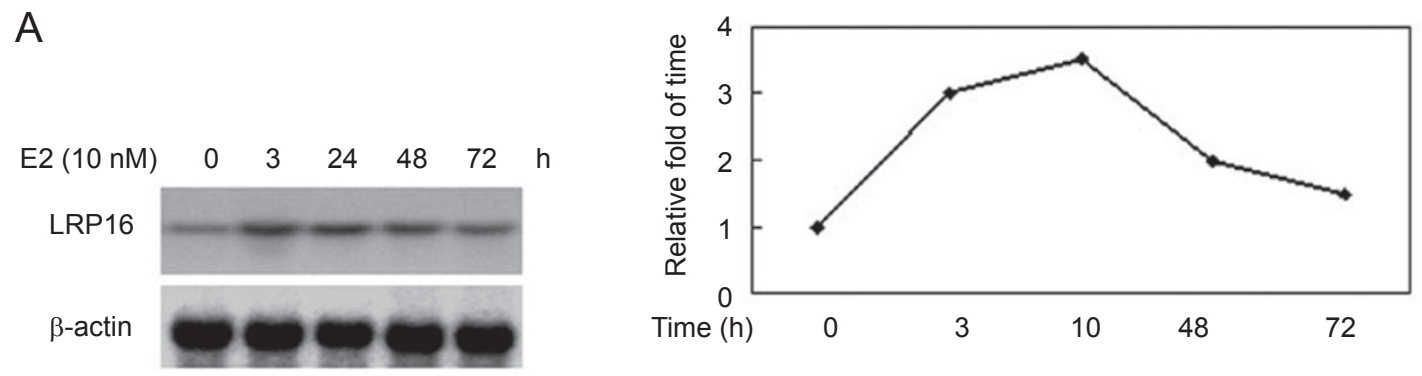

B
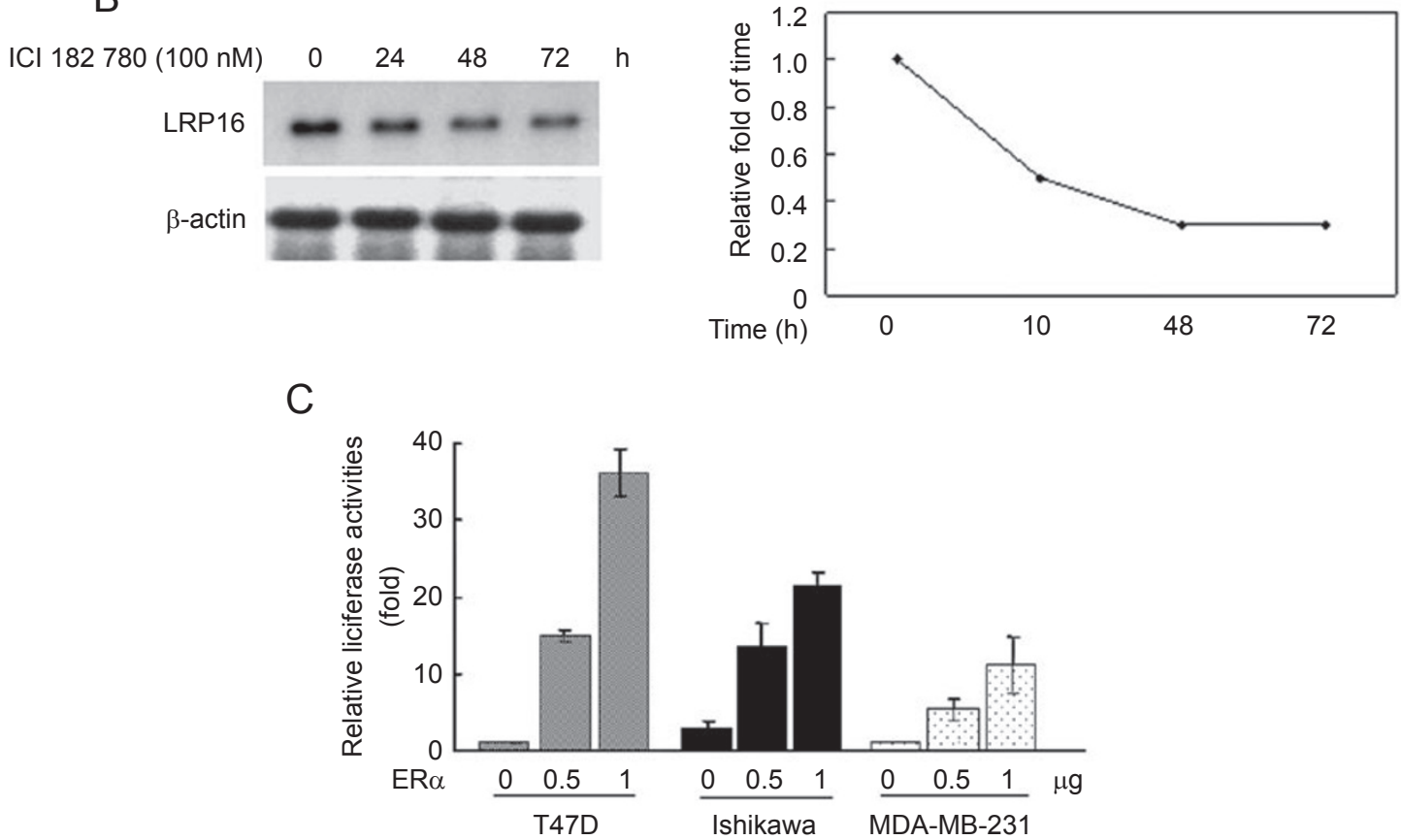

Figure 1 Effect of E2 on transcriptional regulation of LRP16 via ERa in Ishikawa cells. (A, B) After northern blot analyses, data were quantified using the HIHimage/ImageJ software and were corrected for $\beta$-actin. Representative results of multiple repetitions are shown. (C) Cells were co-transfected with the indicated LRP16-luciferase reporter pGLS ${ }_{5}(1 \mu \mathrm{g}), \mathrm{pRL}-\mathrm{SV} 40$ (1 ng) and different amounts of ER $\alpha$ cDNA. Thirty hours after co-transfection, cells were treated with $10 \mathrm{nM}$ E2 for an additional $12 \mathrm{~h}$ and were then harvested. Luciferase values (normalized) for $\mathrm{pGLS}_{5}$ and pRL-SV40 transfection were arbitrarily assigned a value of 1 . These results are expressed as the mean \pm SEM for at least three separate determinations for each treatment group. 
Overexpression of LRP16 does not significantly promote the proliferation of Ishikawa cells

To determine whether the overexpression of LRP16 promotes the proliferation of Ishikawa cells, the pcDNA3.1LRP16 was stably transfected into Ishikawa cells. The empty vector was used as a negative control. The cells were then treated with $1 \mathrm{mg} / \mathrm{ml} \mathrm{G} 418$ for 3 weeks. All of the Ishikawa parental cells were killed within this period. The results from western blot analysis show an approximate three-fold increase of LRP16 protein in Ishikawa cells transfected with ectopic LRP16 over those transfected with the empty vector (Figure 2A). In response to the overexpression of LRP16, we did not observe significant alterations in cell proliferation (Figure 2B).
Overexpression of LRP16 stimulates in vitro invasion of Ishikawa cells

The previous clinicopathological data that LRP16 overexpression is positively linked to the involvement of axillary lymphoid nodes in primary breast carcinomas promoted us to investigate whether the overexpression of LRP16 induces the invasive capacity of Ishikawa cells. To this end, a modified Boyden chamber assay (Transwell assay) was carried out to determine the ability of the cells to invade through biological matrices in vitro. The relevance of this assay for cell invasion and for in vivo malignancy has been documented extensively [27]. Ishikawa is a poorly invasive cell line, but its invasion potential moderately increased in the presence of fibronectin as the chemoattrac-
A

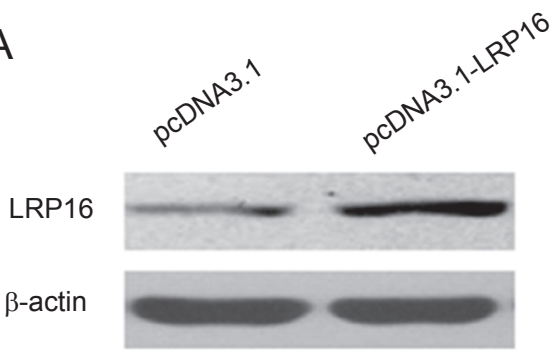

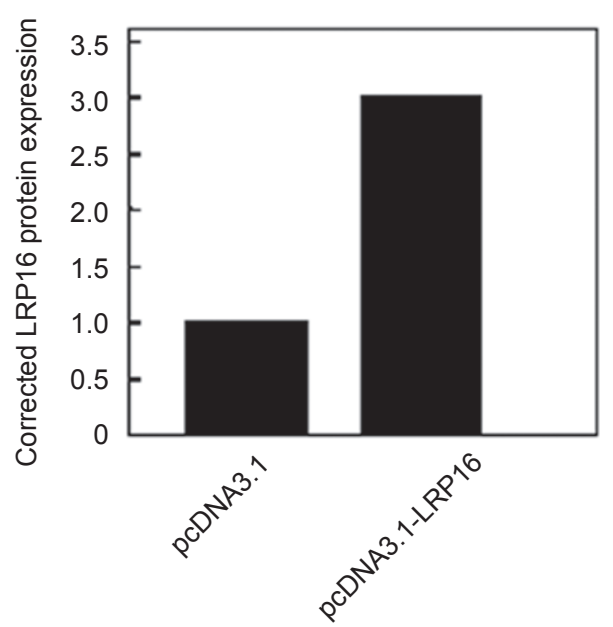

B

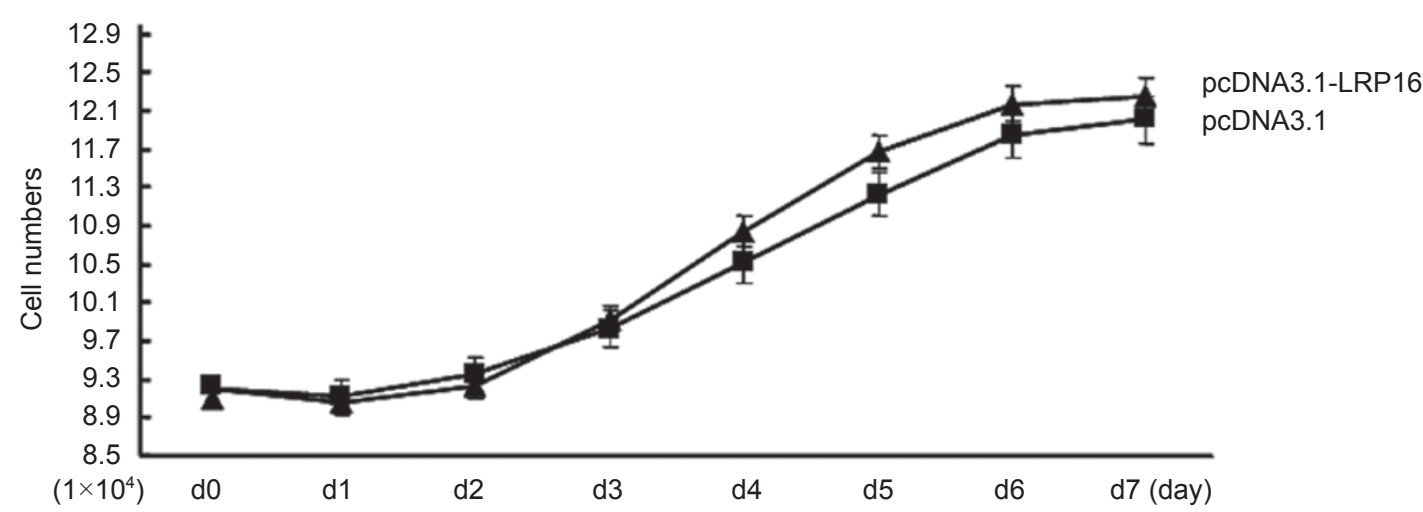

Figure 2 Effect of LRP16 overexpression on proliferation of Ishikawa cells. (A) Immunoblot analyses were probed for LRP16 using polyclonal anti-LRP16 antibodies. $\beta$-Actin was used as a loading control. These representative results were quantified by densitometric scanning and analyzed with the NIHimage/ImageJ software and were corrected for $\beta$-actin. (B) A logarithmic population of the Ishikawa cells expressing either a blank vector or pcDNA3.1-LRP16. Each data point represents the mean \pm SEM number of cells counted in triplicate dishes. 
tant. The cells with or without ectopic LRP16 expression were equally seeded in the upper chamber, whereas, when the number of cells that invaded the Matrigel and attached to the lower filter was calculated, the invasion number of cells with ectopic LRP16 expression was more than that of the control cells (Figure 3A). As shown in Figure 3B, an increase of $30 \%$ in the invasion number of Ishikawa cells was repeatedly observed. These results demonstrate that LRP16 increases the in vitro invasion capacity of Ishikawa cells.

\section{LRP16 suppresses the transcriptional activity of E-cadherin via ER $\alpha$ mediation}

A large number of proteins have previously been reported to be involved in the invasive growth of human EC, including CD44, MMP2, MMP-9 and E-cadherin [15, $28,29]$. To screen which molecule is specific for LRP16- induced invasion of Ishikawa cells, the expression levels of these proteins were measured by western blot analysis. No significant changes were detected for MMP-2, MMP9 and CD44 proteins (data not shown), but a three-fold decrease of E-cadherin protein was observed in Ishikawa cells that overexpress LRP16 (Figure 4A). Next, the expression level of E-cadherin mRNA was examined through northern blot analysis, and the results showed alterations that are consistent with those observed at the protein level (Figure 4A). Then, the inhibitory effect on E-cadherin expression by LRP16 in Ishikawa cells was confirmed by immunohistochemical staining (Figure 4B). In addition, the mRNA and protein levels of E-cadherin were also determined in stable LRP16-knockdown MCF-7 cells, and the results showed the increased expression of E-cadherin in LRP16-siRNA-expressing cells in contrast to the controlsiRNA-expressing cells (Figure 4C). Consistent with this,
A

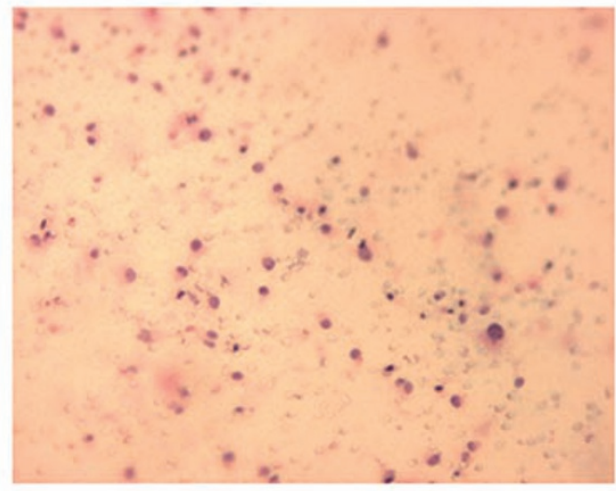

pcDNA3.1

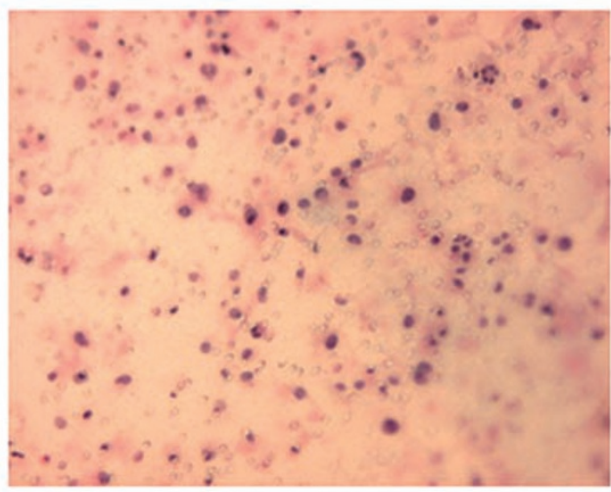

pcDNA3.1-LRP16

B

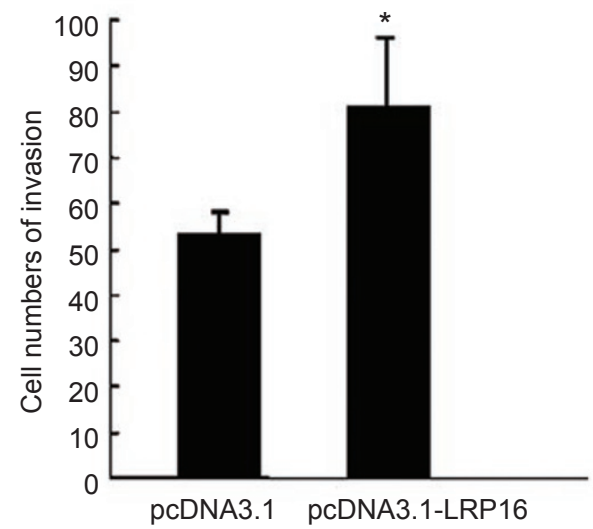

Figure 3 Effect of LRP16 overexpression on the in vitro invasion of Ishikawa cells. (A) Representative pictures of Transwell assays, performed for the indicated cells as described in 'Materials and Methods'. (B) Results are representative of three independent trials. Error bars represent the SEM. Asterisks indicate a significant difference at $p<0.05$. 
A

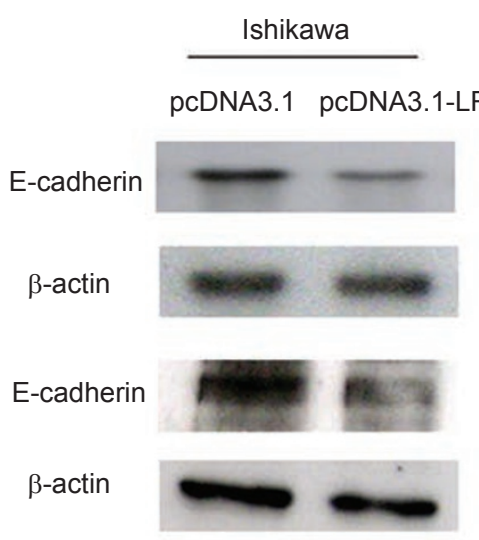

C

Western blot

Northern blot

B

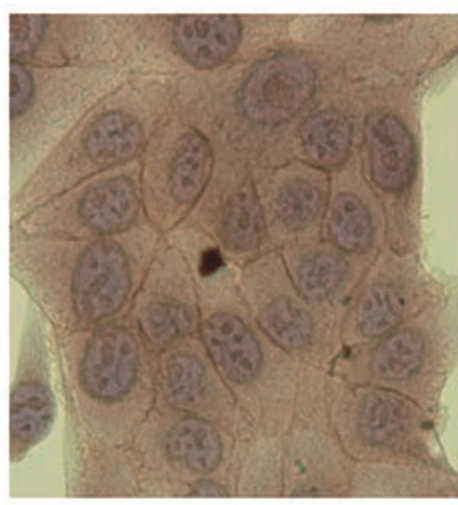

pcDNA3.1

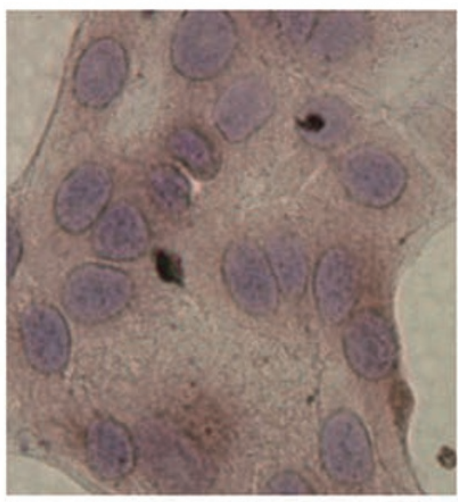

pcDNA3.1-LRP16
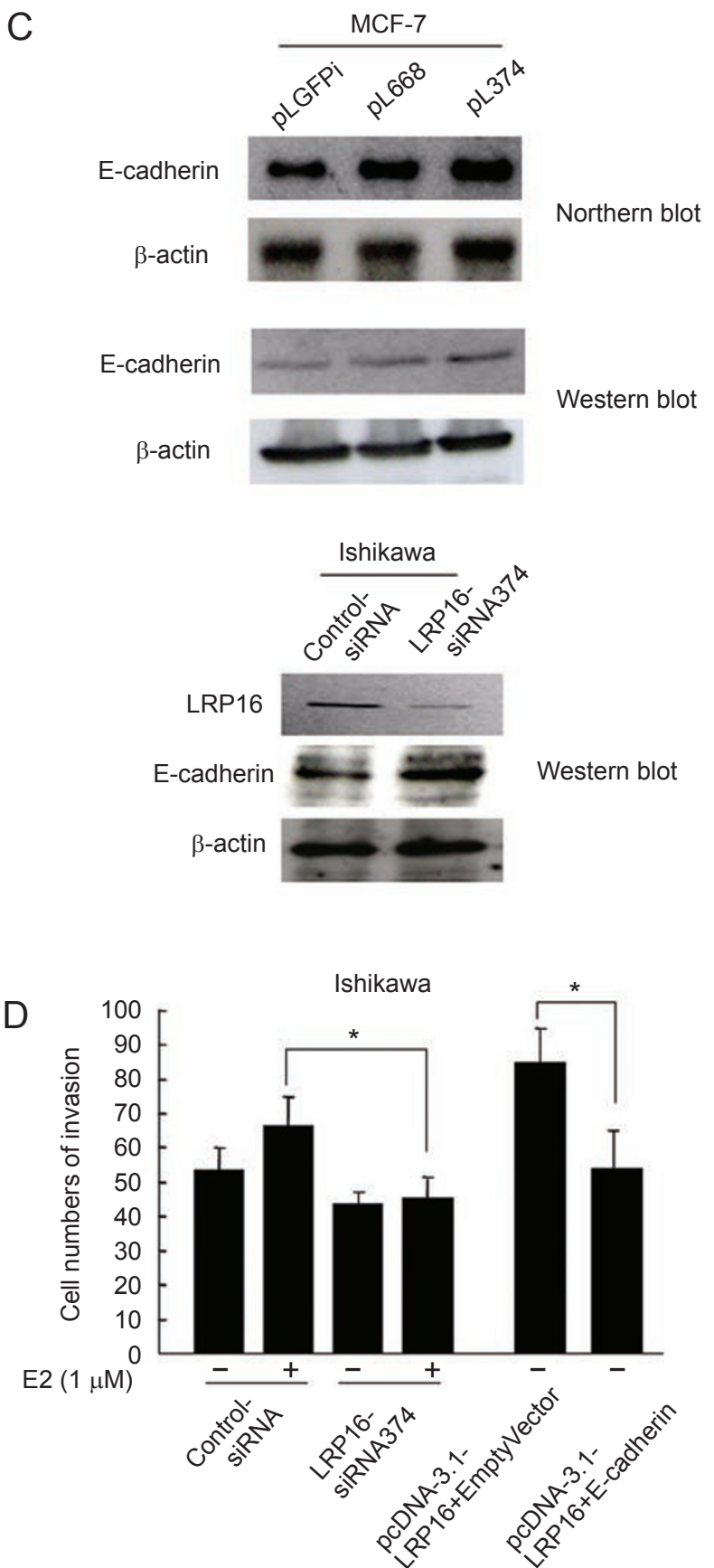

Figure 4 LRP16 suppresses the expression of the E-cadherin gene in Ishikawa cells and MCF-7 cells. The representative results of repeated experiments are shown in (A-C). (A) The mRNA and protein levels of E-cadherin expression were analyzed in Ishikawa cells transfected with the indicated vectors by northern and western blot analyses. $\beta$-Actin was used as a loading control. (B) The cellular distribution of Ecadherin was analyzed in Ishikawa cells transfected with the LRP16 expression vector or the empty vector by immunocytochemistry. (C) pLGFPi, pL374 and pL668 are MCF-7 cells with different expression levels of LRP16 as described in 'Materials and Methods'.pL374 and pL668 cells are LRP16-knockdown cells with stably expressing LRP16-siRNA 374 and LRP16-siRNA 668 as described previously [25]. The mRNA and protein levels of E-cadherin expression were measured by northern and western blot analyses in LRP16-knockdown MCF-7 cells. pLGFPi cells stably expressing siRNA against green fluorescence protein were used as control cells. The protein levels of LRP16 and E-cadherin were analyzed by western blot analysis in Ishikawa cells transiently transfected with LRP16-siRNA or control-siRNA. $\beta$-Actin was used as a loading control. (D) Transwell assays were performed for the indicated cells treated with or without E2 in the upper chamber as described in 'Materials and Methods'. Results are representative of three independent trials. Error bars represent the SEM. Asterisks indicate a significant difference at $p<0.05$. 
transient transfection of LRP16-siRNA 374 caused a dramatic decrease of LRP16 protein and a two-fold increase of E-cadherin in Ishikawa cells relative to the control siRNA (Figure 4C). These data demonstrate the reverse correlation of the expression levels between LRP16 and the E-cadherin gene in both Ishikawa and MCF-7 cells.

To further confirm the effect of E2-induced upregulation of LRP16 on the invasion capacity of Ishikawa cells, we determined the anti-invasion potential of LRP16-siRNA in E2-responsive Ishikawa cells using Transwell assays. As demonstrated in Figure 4D, knockdown of LRP16 in Ishikawa cells decreases the cells' invasion regardless of whether E2 is added in the upper chamber. Furthermore, to test whether the induction of the cells' invasion capacity by LRP16 overexpression is attributed to the downregulation of E-cadherin, the E-cadherin expression vector was transiently transfected in LRP16-overexpressing Ishikawa cells. After $48 \mathrm{~h}$, Ishikawa cells transfected with E-cadherin or pcDNA3.1 empty plasmid were trypsinized and subjected to Transwell assays. The results demonstrate that the ectopic expression of E-cadherin in Ishikawa cells significantly blocks the invasion potential induced by LRP16 (Figure 4D). In general, these results confirm that the E2 induction of LRP16 promotes the Ishikawa cell invasion that is mediated by the downregulation of E-cadherin.

To determine whether LRP16 is a repressor for transcriptional activity of the E-cadherin gene, the E-cadherin promoter construct and LRP16 cDNA were co-transfected into Ishikawa cells. A number of previous studies have suggested that the proximal E-cadherin promoter confers the transcriptional inactivation of E-cadherin in some cancer cell lines [16-18,26]. We decided to use the most proximal human E-cadherin promoter construct (EcadK1-Luc, -108 to +125 ), which has previously been shown to confer the specific epithelial expression to a reporter gene [26, 30]; however, it does not contain any classical EREs. The results show that E-cadK1-Luc activities are efficiently repressed by LRP16 in a dose-dependent manner (Figure 5A). These data demonstrate that LRP16 represses the expression of the E-cadherin gene by inhibiting its transactivation.
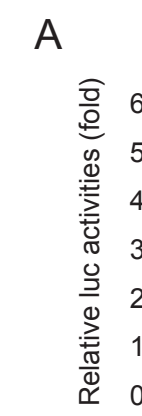

$\operatorname{LRP} 16(\mu \mathrm{g})$

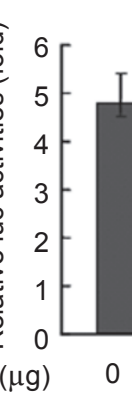

Ishikawa

C

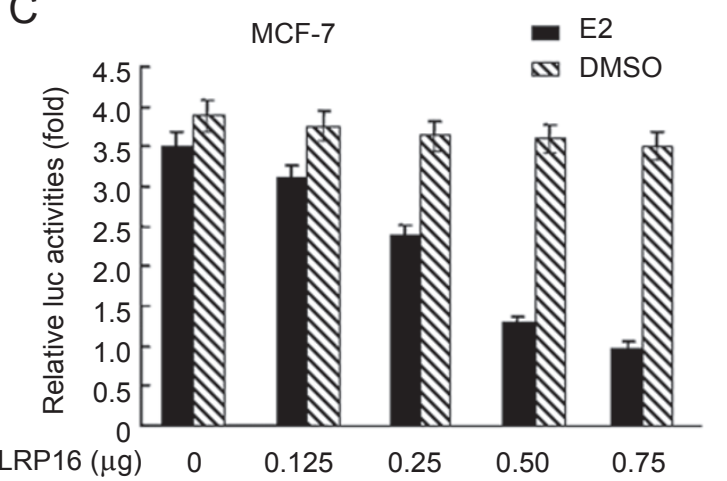

B

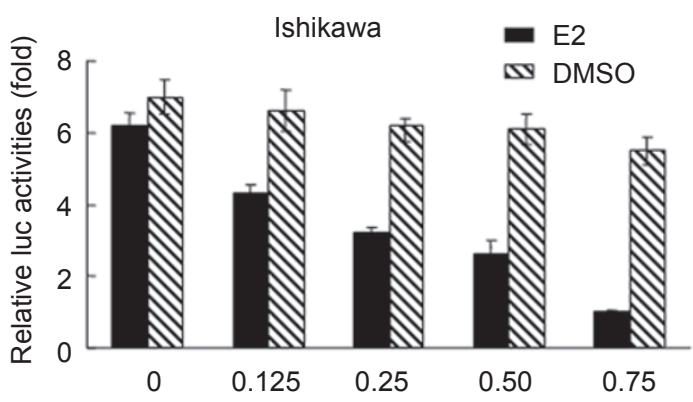

D

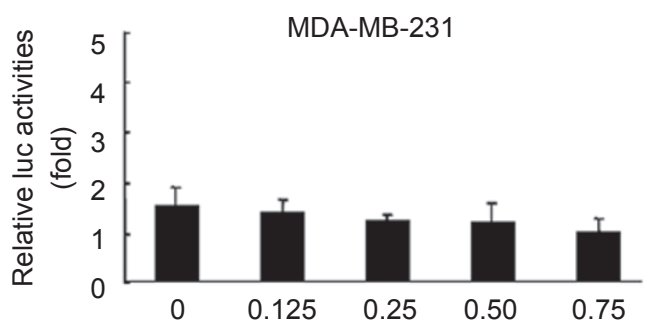

Figure 5 The inhibitory effect on the transactivation of the E-cadherin promoter by LRP16 is dependent on estrogen activity. (A) Ishikawa cells were grown in normal medium, then transfected with E-cadK1-Luc and the effector molecule LRP16. (B-D) Ishikawa and MCF-7 cells were cultured in phenol-red-free medium stripped of steroids. Thirty hours after co-transfection, cells were treated with $10 \mathrm{nM}$ E2 or DMSO. MDA-MB-231 cells were cultured in L15 medium. Luciferase values (normalized) for LRP16 $(0.75 \mu \mathrm{g})$ transfection in the presence of E2 were arbitrary assigned a value of 1 . These results are expressed as the mean \pm SEM for at least three separate determinations for each treatment group. 


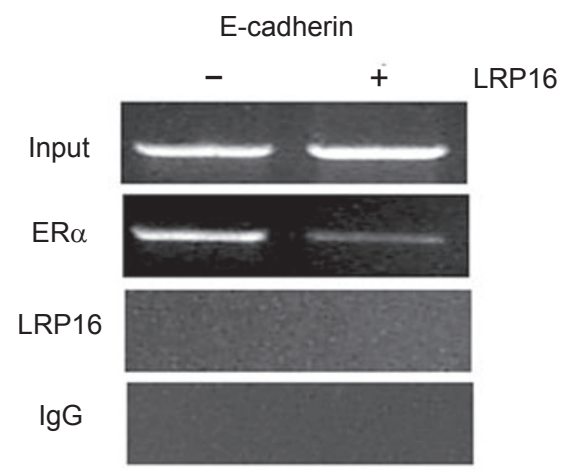

Figure 6 Effect of LRP16 on the recruitment of ER $\alpha$ to the E2-responsive E-cadherin gene promoter. Ishikawa cells were transiently co-transfected with the indicated constructs, treated for $3 \mathrm{~h}$ with 10 nM E2, and subjected to immunoprecipitation and PCR as described in 'Materials and Methods'. Representative results from repeated experiments are shown here.

To further investigate the regulatory mechanism of E-cadherin expression by LRP16, the mRNA levels of transcription factors including Snail, Slug and SIP1, which have been previously well-documented to be repressors of E-cadherin gene [16, 26, 31], were first examined by northern blot analysis. No significant difference was observed between LRP16-overexpressing and control Ishikawa cells (data not shown). Also, it has been documented that ER $\alpha$ downregulates E-cadherin expression through E2-induced interactions of ER $\alpha$ and its co-repressors with the E2-responsive region of the E-cadherin promoter in breast cancer cells [18]. To test whether the repression of E-cadherin transactivation by LRP16 is mediated through the ligandbound ER $\alpha$, we measured the $L u c$ activities by culturing Ishikawa cells in the absence of estrogen and phenol red. As shown in Figure 5B, LRP16 repressed the E-cadK1$L u c$ activities in a dose-dependent manner in the presence of estrogen but not in the absence of estrogen. These data show that the repression effect on the E-cadherin gene transcription by LRP16 is dependent on the estrogen activity. As a further demonstration of specificity, we performed the co-transfection assays in ER $\alpha$-positive MCF-7 and ER $\alpha$-negative MDA-MB-231 human breast cancer cells. The results summarized in Figure 5C and 5D demonstrate that LRP16 inhibits the transcriptional activities of the E-cadherin promoter construct in the presence of E2 in MCF-7 cells, but not in MDA-MB-231 cells.

\section{LRP16 inhibits the recruitment of ER $\alpha$ to the E-cadherin promoter}

To analyze whether the decreased promoter activity of
E-cadherin by LRP16 was the direct result of the recruitment of LRP16 and ER $\alpha$, we performed ChIP assays. EcadK1-Luc, which is known to be repressed by LRP16 in the presence of E2, was co-transfected with the ER $\alpha$ and LRP16 expression vectors into Ishikawa cells. The cells were then treated with E2 $(10 \mathrm{nM})$ for $3 \mathrm{~h}$, and the recruitment of ER $\alpha$ and LRP16 was analyzed by ChIP (Figure 6). In the absence of LRP16 transfection, we repeatedly detected a high level of ER $\alpha$ binding at the promoter of E-cadherin. Upon LRP16 transfection into the cells, ER $\alpha$ was released from the E-cadherin promoter DNA. In both groups we did not detect the presence of LRP16 at the E-cadherin promoter. The results from this ChIP analysis suggest that LRP16 may inhibit E-cadherin transcription by antagonizing ER $\alpha$ binding to the regulatory region of E-cadherin and also suggest a possible interaction of ER $\alpha$ and LRP16.

\section{Discussion}

$\mathrm{EC}$ is the most commonly diagnosed malignancy of the female genital tract. Estrogen and most of the selective estrogen-receptor modulators are believed to be involved in endometrial carcinogenesis through their effects on transcriptional regulation [8]. Therefore, the isolation of the downstream targets of ER in EC cells could lead to the identification of the genes that are involved in the initiation and progression of ECs. Here, we identified the LRP16 gene as a target of transcriptional activation by ER $\alpha$ in ER $\alpha$-positive Ishikawa cells, similar to that in breast cancer cells. In contrast to those reported previously in MCF-7 cells [22], overexpression of LRP16 in Ishikawa cells did not significantly stimulate cell proliferation. These differential effects of LRP16 on cellular proliferation may be attributable to the different cellular context between MCF-7 and Ishikawa cells. Importantly, our results document a novel ER $\alpha /$ LRP16/E-cadherin signaling pathway in this cell culture system, which may be involved in the progression of ER $\alpha$-positive ECs.

The dependence of LRP16 levels on estrogen activity predicts a mechanism by which ER $\alpha$ signaling can affect E-cadherin biosynthesis. ER $\alpha$ directly activates LRP16 transcription in response to estrogen signaling. LRP16, in turn, represses E-cadherin expression in the presence of estrogen signaling. Ultimately, this biological output of ER signaling through LRP16 is manifested in the expression modulation of E-cadherin. The ability of LRP16, whose biosynthesis depends on the estrogen activity, to functionally modulate ER $\alpha$-mediated transcriptional repression contributes both to the complexity and to the plasticity of the regulatory circuit.

Previous studies have linked ER $\alpha$ and E-cadherin 
expression in human breast cancer cell lines and primary breast carcinomas [18, 19, 32, 33]. E-cadherin is often positive in ER $\alpha$-positive cancer cells, and is often negative in ER $\alpha$-negative tumor cells (such as MCF-7 versus MDA-MB-231 cells), and the loss of E-cadherin may result in the more aggressive growth of ER $\alpha$-negative cells by increasing the probability of invasion and metastasis. In our study, we documented the inhibitory effect of LRP16 on E-cadherin expression in ER $\alpha$-positive cancer cells. The different response of the E-cadherin promoter to LRP16 in the presence of E2 between ER $\alpha$-positive and negative tumor cells (Figure 5) confirmed the need for ER $\alpha$ for the ability of LRP16 to inhibit E-cadherin expression. In general, from the cell-culture model, our data establish a novel molecular axis from ER $\alpha$ signaling to E-cadherin expression in ER $\alpha$-positive cancer cells.

The regulation of E-cadherin expression by ER $\alpha$ involves multiple molecular pathways in ER $\alpha$-positive cancer cells. For example, MTA3, an ER $\alpha$ target gene, indirectly induces E-cadherin transcription by directly repressing the expression of Snail, which is a well-documented Ecadherin repressor [19]. Evidence also suggests that the E2-mediated downregulation of E-cadherin involves in the functional interactions of $E R \alpha$ and its co-repressors, such as SAFB and NCoR, with the proximal E-cadherin promoter in MCF-7 cells [18]. Our ChIP analyses also show an involvement of ER $\alpha$ at the E-cadherin promoter in Ishikawa EC cells, but LRP16, unlike SAFB and NCoR, cannot be immunoprecipitated at the E-cadherin promoter (Figure 6). However, in the LRP16-overexpressing cellular context, ERa is partially released from the E-cadherin promoter (Figure 6), indicating that LRP16 has an antagonistic role in the process of ER $\alpha$ binding to the E-cadherin promoter DNA. Several previous studies have suggested that a balance between the co-activators and co-repressors of ER $\alpha$ is an important factor for modulating ER $\alpha$-repressed gene expression, the disruption of which may incur the alteration of receptor binding to the promoter of its target genes $[34,35]$. In addition, several lines of evidence indicate that the ability of nuclear receptors (NRs) to stimulate transcription requires chromatin remodeling and the functional interaction of NRs with chromatin-remodeling complexes [36-38]. As for the detailed mechanism of LRP16 blocking the access of ER $\alpha$ to the E-cadherin promoter, we would like to propose that LRP16 may exert its molecular action by remodeling DNA structure because of a conserved Macro_Appr_pase like motif present in LRP16, which is also found in a number of proteins that are associated with DNA and/or RNA unwinding (available at http://smart.embl-heidelberg.de/smart/do_annotation. $\mathrm{pl}$ ?DOMAIN=A1pp). Experiments are ongoing to test this hypothesis by examining the presence of LRP16 in the chromatin-remodeling complexes and the interactions between LRP16 and ER $\alpha$ or its cofactors.

Metastasis is the most common cause of death in patients with cancer. Our previous study that positively linked the overexpression of LRP16 mRNA in primary breast carcinoma samples with the involvement of axillary lymphoid nodes suggested the involvement of LRP16 during the metastasis of breast cancer [24]. In carcinomas, the metastatic process is thought to consist of a number of distinct steps. Invasion is the first step, which requires tumor cells to lose cell-cell adhesion and to gain motility, and subsequently involves the detachment of cells from the primary tumor and migration of tumor cells through the extracellular matrix $[39,40]$. At this stage, one of the proteins that are downregulated is E-cadherin, a molecule essential in the establishment of homotypic adhesion junctions [13, 41]. A number of experimental and clinical investigations revealed that the loss of E-cadherin expression is associated with endometrial tumor cell invasion and poor prognosis [13, $15,42]$. Dysregulation of several transcription regulators of the E-cadherin gene such as Twist, Snail and progesterone receptor is also associated with the progression of ECs [43-45]. In this study, we document that LRP16 is a novel repressor of E-cadherin. Using a modified Boyden chamber assay, we observed that the ectopic expression of the LRP16 gene significantly promotes the invasive capacity of Ishikawa cells (Figure 3). By contrast, inhibition of the endogenous LRP16 in Ishikawa cells blocked the invasion of E2-stimulated cells (Figure 4D). We also provide evidence that the promoting effect of LRP16 on invasive behavior can be attributed to the ability of LRP16 to suppress the expression of E-cadherin (Figure 4D). Studies analyzing the relationship between ER $\alpha$, LRP16 and E-cadherin expression in large carcinoma samples are ongoing.

Our data represent the first evidence to link ER $\alpha$-mediated transcriptional signaling to E-cadherin expression by the E2-regulated LRP16 gene in Ishikawa human EC cells, implying a novel mechanism of estrogen-induced progression of EC. Although the in vivo function of LRP16 is unknown, the increased invasion capacity of Ishikawa cells following LRP16 overexpression suggests an important role in the progression of EC. If similar results could be reproduced in vivo using an athymic mouse model and a positive link could be established between LRP16 overexpression and unfavorable prognosis in patients with EC, then the suppression of LRP16 expression may significantly prolong the survival of patients with EC by inhibiting or slowing down the invasive capacity of EC cells. The identification of the ER $\alpha / \mathrm{LRP} 16 / \mathrm{E}$-cadherin signaling pathway may provide a potential target for anti-estrogen therapy and prevention of invasion. 


\section{Acknowledgments}

We sincerely thank Dr Pudi Renuka and Dr Jianjun Zhou at the National Cancer Institute for their critical reading of our manuscript. We thank Dr Eric R Fearon at the University of Michigan in USA for the E-cadherin promoter reporters and Dr Nowata at Kyushu University of Japan for the ER $\alpha$ expression vector. This work was supported by the National Natural Science Foundation of China (30471813, 30572096 and 30670809), the Beijing Natural Science Foundation of China (5052024 and 7052061) and the Chinese PLA National Science Fund for Distinguished Young Scholars grant (06J017).

\section{References}

1 Nillsson S, Makela S, Treuter E, et al. Mechanisms of estrogen action. Physiol Rev 2001; 81:1535-1565.

2 McDonnell DP, Norris JD. Connections and regulation of the human estrogen receptor. Science 2002; 296:1642-1644.

3 DeNardo DG, Kim HT, Hilsenbeck S, Cuba V, Tsimelzon A, Brown PH. Global gene expression analysis of estrogen receptor transcription factor cross talk in breast cancer: identification of estrogen-induced/activator protein-1-dependent genes. Mol Endocrinol 2005; 19:362-378.

4 Safe S. Transcriptional activation of genes by $17 \beta$-estradiol through estrogen receptor-Sp1 interactions. Vitam Horm 2001; 62:231-252.

5 Shang Y, Brown M. Molecular determinants for the tissue specificity of SERMs. Science 2002; 295:2465-2468.

6 Emons G, Fleckenstein G, Hinney B, et al. Hormonal interactions in endometrial cancer. Endocr Relat Cancer 2000; 7:227-242.

7 Ryan AJ, Susil B, Jobling TW, Oehler MK. Endometrial cancer. Cell Tissue Res 2005; 322:53-61.

8 Shang Y. Molecular mechanisms of oestrogen and SERMs in endometrial carcinogenesis. Nat Rev Cancer 2006; 6:360-368.

9 Feng ZY, Shiozawa T, Miyamoto T, et al. Overexpression of hedgehog signaling molecules and its involvement in the proliferation of endometrial carcinoma cells. Clin Cancer Res 2007; 13:1389-1398.

10 Chen CL, Hsieh FC, Lieblein JC, et al. Stat3 activation in human endometrial and cervical cancers. Br J Cancer 2007; 96:591599.

11 Haughian JM, Jackson TA, Koterwas DM, Bradford AP. Endometrial cancer cell survival and apoptosis is regulated by protein kinase C alpha and delta. Endocr Relat Cancer 2006; 13:12511267.

12 Gumbiner BM. Cell adhesion: the molecular basis of tissue architecture and morphogenesis. Cell 1996; 84:345-357.

13 Perl AK, Wilgenbus P, Dahl U, Semb H, Christofori G. A causal role for E-cadherin in the transition from adenoma to carcinoma. Nature 1998; 392:190-193.

14 Hanahan D, Weinberg RA. The hallmarks of cancer. Cell 2000; 100:57-70.

15 Mell LK, Meyer JJ, Tretiakova M, et al. Prognostic significance of E-cadherin protein expression in pathological stage I-III endometrial cancer. Clin Cancer Res 2004; 10:5546-5553.
16 Batlle E, Sancho E, Franci C, et al. The transcription factor Snail is a repressor of $E$-cadherin gene expression in epithelial tumour cells. Nat Cell Biol 2000; 2:84-89.

17 Yang J, Mani SA, Donaher JL, et al. Twist, a master regulator of morphogenesis, plays an essential role in tumor metastasis. Cell 2004; 117:927-939.

18 Oesterreich S, Deng W, Jiang S, et al. Estrogen-mediated downregulation of E-cadherin in breast cancer cells. Cancer Res 2003; 63:5203-5208.

19 Fujita N, Jaye DL, Kajita M, et al. MTA3, a Mi-2/NuRD complex subunit, regulates an invasive growth pathway in breast cancer. Cell 2003; 113:207-219.

20 Han WD, Yu L, Lou FD, et al. Cloning and expression characterization of the full-length cDNA for a novel leukemia-associated gene LRP16. Chin J Biochem Mol Biol 2001; 17:209-214.

21 Han WD, Lou FD, Yu L, et al. Bioinformatic analysis and subcellular distribution of LRP16 protein. Acad J PLA Postgrad Med School 2002; 23:277-279.

22 Han WD, Mu YM, Lu XC, et al. Up-regulation of LRP16 mRNA by $17 \beta$-estrodial through activation of estrogen $\alpha(E R \alpha)$, but not ER $\beta$, and promotion of human breast cancer MCF-7 cell proliferation: a preliminary report. Endocr Relat Cancer 2003; 10:217-224.

23 Zhao YL, Han WD, Li Q, et al. Mechanism of transcriptional regulation of LRP16 gene expression by $17-\beta$ estradiol in MCF-7 human breast cancer cells. J Mol Endocrinol 2005; 34:77-89.

24 Liao DX, Han WD, Zhao YL, et al. The expression and clinical significance of LRP16 gene in human breast cancer. Ai Zheng 2006; 25:44-48

25 Han WD, Zhao YL, Li Q, et al. Inhibition of proliferation of human breast cancer MCF-7 cells by small interference RNA against LRP16 gene. Chin J Cancer Res 2004; 16:239-245.

26 Hajra KM, Chen DY, Fearon ER. The SLUG zinc-finger protein represses E-cadherin in breast cancer. Cancer Res 2002; 62:16131618.

27 Hendrix MJ, Seftor EA, Seftor ER, Fidler IJ. A simple quantitative assay for studying the invasive potential of high and low human metastatic variants. Cancer Lett 1987; 38:137-147.

28 Leblanc M, Poncelet C, Soriano D, et al. Alternation of CD44 and cadherin expression: possible association with augmented aggressiveness and invasiveness of endometrial carcinoma. Virchows Arch 2001; 438:78-85.

29 Aglund K, Rauvala M, Puistola U, et al. Gelatinases A and B (MMP-2 and MMP-9) in endometrial cancer-MMP-9 correlates to the grade and the stage. Gynecol Oncol 2004; 94:699-704.

30 Hajra KM, Ji X, Fearon ER. Extinction of E-cadherin expression in breast cancer via a dominant repression pathway acting on proximal promoter elements. Oncogene 1999; 18:7274-7279.

31 Comijn J, Berx G, Vermassen P, et al. The two-handed E-box binding zinc finger protein SIP1 downregulates E-cadherin and induces invasion. Mol Cell 2001; 7:1267-1278.

32 Siitonen SM, Kononen JT, Helin HJ, et al. Reduced E-cadherin expression is associated with invasiveness and unfavorable prognosis in breast cancer. Am J Clin Pathol 1996; 105:394-402.

33 Lipponen P, Saarelainen E, Ji H, Aaltomaa S, Syrjanen K. Expression of E-cadherin (E-CD) as related to other prognostic factors and survival in breast cancer. J Pathol 1994; 174:101-109.

34 Zubairy S, Oesterreich S. Estrogen-repressed genes - key mediators of estrogen action? Breast Cancer Res 2005; 7:163-164. 
35 Newman SP, Bates NP, Vernimmen D, Parker MG, Hurst HC. Cofactors competition between the ligand-bound oestrogen receptor and an intron 1enhamcer leads to oestrogen repression of ERBB2 expression in breast cancer. Oncogene 2000; 19:490497.

36 Belandia B, Parker MG. Nuclear receptors: a rendezvous for chromatin remodeling factors. Cell 2003; 114:277-280.

37 Nuclear receptor mediated gene regulation through chromatin remodeling and histone modifications. Endocr J 2006; 53:157172.

38 Kinyamu HK, Archer TK. Modifying chromatin to permit steroid hormone receptor-dependent transcription. Biochem Biophys Acta 2004; 1677:30-45.

39 Woodhouse EC, Chuaqui RF, Liotta LA. General mechanisms of metastasis. Cancer 1997; 80:1529-1538.

40 Cavallaro U, Christofori G. Cell adhesion in tumor invasion and metastasis: loss of the glue is not enough. Biochim Biophys Acta 2001; 1552:39-45.
41 Beavon IR. The E-cadherin-catenin complex in tumour metastasis: structure, function and regulation. Eur J Cancer 2001; 36:1607-1620.

42 Saito T, Nishimura M, Yamasaki H, Kudo R. Hypermethylation in promoter region of E-cadherin gene is associated with tumor dedifferention and myometrial invasion in endometrial carcinoma. Cancer 2003; 97:1002-1009.

43 Kyo S, Sakaguchi J, Ohno S, et al. High twist expression is involved in infiltrative endometrial cancer and affects patient survival. Hum Pathol 2006; 37:431-438.

44 Yoshida H, Broaddus R, Cheng W, Xie S, Naora H. Deregulation of the HOXA10 homeobox gene in endometrial carcinoma: role in epithelial-mesenchymal transition. Cancer Res 2006; 66:889897.

45 Hanekamp EE, Gielen SC, Smid-Koopman E, et al. Consequences of loss of progesterone receptor expression in development of invasive endometrial cancer. Clin Cancer Res 2003; 9:4190-4199. 